\title{
Evaluation of Diagnosis and Management of Omega-5-Gliadin Allergy: A Retrospective Survey
}

\author{
Celia Zubrinich (D) ${ }^{1,2}$ \\ Robert Puy (D) ${ }^{\text {I,3 }}$ \\ Robyn O'Hehir (D) ${ }^{1,3}$ \\ Mark Hew ${ }^{1,2}$ \\ 'Allergy, Asthma \& Clinical Immunology, \\ Alfred Health, Melbourne, Victoria, \\ Australia; ${ }^{2}$ School of Public Health \& \\ Preventive Medicine, Monash University, \\ Melbourne, Victoria, Australia; ${ }^{3}$ Allergy, \\ Immunology and Respiratory Medicine, \\ Central Clinical School, Monash \\ University, Melbourne, Victoria, Australia
}

Correspondence: Celia Zubrinich Email c.zubrinich@alfred.org.au
Background: Allergy to the omega-5-gliadin component of gluten (O-5-G allergy) often manifests when wheat ingestion is followed by a co-factor, usually exercise. There is no established best approach to management.

Objective: We sought to identify the beneficial effects, firstly of establishing a firm diagnosis, and secondly of stringent management, either by avoiding gluten ingestion altogether or separating it temporally from exercise by at least 4 hours. We also determined how frequently patients adhered to their physicians' clinical recommendations.

Methods: We undertook a survey of individuals diagnosed with O-5-G allergy at our institution over 8 years, who had a consistent clinical history and confirmatory laboratory evidence.

Results: Of 80 eligible individuals, 43 responded (54\%). Symptoms began in adulthood for all bar one, and concurrent asthma and eczema was uncommon $(9 \%$ prevalence, respectively). Median time to diagnosis was 2 years. Achieving a diagnosis reduced the rate of reactions $(0.35$ per month vs 1.085 reactions per month, $p=0.029)$. Many patients $(10 / 43)$ did not adhere to the recommended stringent approach, to either avoid wheat/gluten or separate food and exercise by 4 hours. However, those adopting a stringent approach had a substantially lower risk of recurrent allergic reaction $(0.22$ per month vs 0.74 per month, $\mathrm{p}=0.004)$.

Conclusion: The epidemiology of $\mathrm{O}-5-\mathrm{G}$ allergy implies pathogenic mechanisms potentially distinct from those of childhood-onset food allergy. Accurate diagnosis improves the clinical trajectory, primarily through the adoption of a stringent management approach.

Keywords: omega-5-gliadin allergy, wheat allergy, food allergy, anaphylaxis, wheatdependent exercise-induced anaphylaxis, WDEIA, food-dependent exercise-induced anaphylaxis, FDEIA

\section{Introduction}

Food-dependent exercise-induced anaphylaxis (FDEIA) is a disorder whereby anaphylaxis occurs if a patient exercises within a few hours of ingesting a specific food to which they are sensitized. Wheat is the most common food associated with this condition as Wheat Dependent Exercise Induced Anaphylaxis (WDEIA) and has been the most studied. Of the wheat allergens, omega-5-gliadin (O-5-G), a protein component of gluten, has been implicated as the most frequent trigger of WDEIA although other wheat allergens have been identified. ${ }^{1-3}$ Although exercise is the key co-factor, other known co-factors include alcohol, menstruation and aspirin/NSAIDs. ${ }^{4}$ This unusual condition was first recognised decades ago ${ }^{5}$ but remains less well characterized than many other food allergies. 
The necessary conjunction of wheat ingestion and exercise required to trigger a reaction may escape the notice of patients and clinicians for many months or even years, delaying the diagnosis and potentially increasing the risks of reaction. ${ }^{6}$ Once clinically suspected, however, the diagnosis of $\mathrm{O}-5-\mathrm{G}$ allergy in most cases is readily confirmed by positive serum-specific IgE against $\mathrm{O}-5-\mathrm{G} .^{7}$

Omega-5-gliadin allergy (O-5-G allergy) is clearly a manifestation of wheat allergy, so patients are often advised that the safest course is to entirely avoid wheat or gluten. ${ }^{2}$ Others rely on separating wheat ingestion from exercise by at least 4 hours to protect them from reactions. ${ }^{6}$ The optimal management approach remains unclear, and it is also not reported how frequently patients adhere to clinical recommendations.

In this retrospective real-world study, we examined the effects on allergic reaction rates of firstly, establishing a firm diagnosis of O-5-G allergy, and secondly, stringently avoiding gluten ingestion altogether or separating gluten ingestion temporally from exercise. We also determined how frequently patients adhered to their doctors' clinical recommendations.

\section{Methods}

\section{Patient Cohort}

Our tertiary university academic centre, Alfred Health, Melbourne, Victoria, Australia, provides allergy services for the surrounding metropolitan area and additionally draws referrals from the entire state of Victoria, Australia, providing approximately 6000 outpatient consultations per year. The hospital pathology laboratory performs testing for serum-specific $\mathrm{IgE}$ to $\mathrm{O}-5-\mathrm{G}$ using ImmunoCAP $^{\circledR}$ assay (Phadia, Uppsala, Sweden).

Adults with a diagnosis of O-5-G allergy were identified through a review of positive pathology results between 1 November 2008 (when the test first became available locally) and 31 December 2016. Medical records were reviewed subsequently, to confirm a definite clinical history of a systemic allergic reaction in the setting of wheat or gluten ingestion as well as a relevant co-factor, mostly exercise. Patients were included in the study only if they had a definite clinical history of WDEIA AND a positive serum-specific IgE to $\mathrm{O}-5-\mathrm{G}(>0.35 \mathrm{kUA} / \mathrm{L})$.

\section{Survey}

A paper questionnaire was posted to the patients' home addresses, and in the event of no response, a second questionnaire was posted within 6 months. The questionnaire elicited (full details in Supplement) patient demographics and self-reported atopic co-morbidities- classed as asthma, eczema, "hayfever", or additional food allergies; the interval from O-5-G allergy symptom onset to diagnosis, the time since diagnosis, and the number of allergic episodes during each of those periods; dietary and exercise strategies, and other aspects of management that were recommended by their allergist; treatment strategies that respondents actually adopted into their routine; and self-identified contributing factors to allergic reactions. The rate of allergic events prior to and subsequent to diagnosis was calculated for the respective time periods. The atopic status was assessed by positive results against common inhalant allergens (as serum IgE or skin prick testing) and any measured serum tryptases were reviewed to identify any underlying mast cell disorders. To address the diversity of possible combinations of dietary and exercise strategies, we defined a stringent management approach as one that required complete avoidance of wheat/gluten ("avoiders") or separation of food and exercise by a minimum of 4 hours ("separators"). A liberal management approach involved neither of these.

We stated that responses would be de-identified, and thus the return of a completed questionnaire implied consent, in accordance with the Declaration of Helsinki. Ethics board approval was obtained for this study (Alfred Health REB No. 279/14). Statistics were calculated using $t$-tests in SPSS (Version 25).

\section{Results}

\section{Respondent Demographics}

Laboratory and medical records identified 80 individuals over the 8-year study period with clinical histories consistent with O-5-G allergy and a positive serum-specific O-5-G IgE, indicating this diagnosis is made infrequently. Questionnaires were sent to all 80. Of these, 37 responded and six were "returned to sender" (RTS). A second mail out to the remaining 37 yielded six further responses giving an overall response rate of 43/74 (58\%). Characteristics of the survey respondents are shown in Table 1.

All respondents were adults (age $>18$ years), and all but one had the first onset of symptoms in adulthood. Men and women were evenly represented. In comparison, the 37 non-returned surveys represented an age range 21-82 years and comprised 23 males and 14 females. 
Table I Demographics and Clinical Features of 43 Individuals with Omega-5-Gliadin Allergy

\begin{tabular}{|c|c|}
\hline Age, Mean (Range) & 45 Yrs $(21-76)$ \\
\hline Sex (Male:Female) & 22:21 \\
\hline Asthma, n (\%) & $4(9)$ \\
\hline Eczema, n (\%) & $4(9)$ \\
\hline Other food allergies, $n$ (\%) & $20(47)$ \\
\hline No self-reported atopic comorbidities, $n(\%)$ & $17(40)$ \\
\hline Confirmed atopic status, n (\%) & $23(53)$ \\
\hline Age of symptom onset, mean (range) & 37.2 yrs $(16.8-65.7)$ \\
\hline Symptom onset to diagnosis, median (range) & 2 yrs (I mth-30 yrs) \\
\hline Anaphylaxis ever, $\mathrm{n}(\%)$ & $38(88)$ \\
\hline Epinephrine autoinjector, $\mathrm{n}(\%)$ & $39(91)$ \\
\hline Dietitian review, n (\%) & $13(30)$ \\
\hline
\end{tabular}

\section{Atopic and Allergic History}

The most common self-reported atopic comorbidity was an additional food allergy (20 respondents, 47\%). Asthma and eczema were both uncommon. No reported atopic comorbidities were reported by 17 respondents $(40 \%)$, see Table 1 . Atopic status according to skin prick testing or serum $\operatorname{IgE}$ results was available in 31 individuals and confirmed in 23/ $43(53 \%) .8 / 43(19 \%)$ could be defined as nonatopic and the status was unknown in $12 / 43(28 \%)$. A random serum tryptase was measured contemporaneously with the $\operatorname{IgE}$ testing in all but one individual, with a mean of $4.26 \mathrm{mcg} /$ L, Range 1.1-13.1. (Normal Range $0-11.4 \mathrm{mcg} / \mathrm{L}$ ) Although three individuals had documentation of acutely elevated tryptase in the setting of anaphylaxis, only one individual had an elevation of random tryptase. Follow-up was not undertaken regarding this so a mast cell disorder had not been excluded. The majority of respondents $(38 / 43,88 \%)$ reported having experienced anaphylaxis at least once.

\section{Reactions Before Diagnosis}

The mean allergic reaction rate prior to diagnosis was 1.085 episodes per month. Time between symptom onset and diagnosis ranged from 1 month (single reaction diagnosed at first clinic visit) to 30 years, with a median of 2 years. Thirteen respondents $(30 \%)$ had remained undiagnosed for longer than 5 years.

The time between diagnosis and completing the questionnaire (ie, the time each respondent had lived with knowledge of the diagnosis) ranged from 1 month to 13 years, with a median of 2 years.

\section{Recommended Treatment Strategies (Table 2)}

Most respondents, 39/43 (91\%), had been prescribed an adrenaline auto-injector and 15/43 (35\%) reported having an Action Plan (there were four non-responses).

Respondents reported receiving a range of dietary advice. Thirty-three respondents (77\%) were recommended stringent avoidance by completely or mostly avoiding ingestion of wheat or gluten. Other advice was to exclude wheat/gluten occasionally (seven respondents, $16 \%$ ). One reported advice to avoid wheat around half the time and one reported advice to avoid no foods. One did not respond to this question. Only 13 respondents $(30 \%)$ reported a consultation with a dietitian.

Respondents also reported receiving a range of recommendations regarding exercise. A stringent approach was recommended to either separate wheat/gluten ingestion and exercise by at least 4 hours (16 respondents, 37\%) or to separate eating of any food and exercise by at least 4 hours (13 respondents, 30\%). Other advice was to exercise without restriction (12 respondents, 28\%). Two did not respond to this question.

All but 5 individuals (12\%) reported having been given advice consistent with a stringent management approach, so that of the 12 who received advice to exercise without restriction, 7 were also advised to do so along with a completely wheat-/gluten-free diet.

Table 2 Recommended versus Adopted Treatment Approaches Among 43 Individuals with Omega-5-Gliadin Allergy

\begin{tabular}{|c|c|c|}
\hline & \multicolumn{2}{|c|}{ Separation from Exercise “Separators" } \\
\hline & Yes & No \\
\hline \multicolumn{3}{|c|}{$\begin{array}{l}\text { Dietary Restriction } \\
\text { "Avoiders" }\end{array}$} \\
\hline Yes & $\begin{array}{l}* 11 \\
* * 3\end{array}$ & $\begin{array}{l}* 10 \\
* * 10\end{array}$ \\
\hline $\begin{array}{l}\text { Diet } \\
\text { "Avc }\end{array}$ & & \\
\hline No & $\begin{array}{l}* 17 \\
* * 20\end{array}$ & $\begin{array}{c}* 2 \\
* * 10\end{array}$ \\
\hline
\end{tabular}

Notes: *Recommended measures $(n=40)$. **Adopted measures $(n=43)$. 


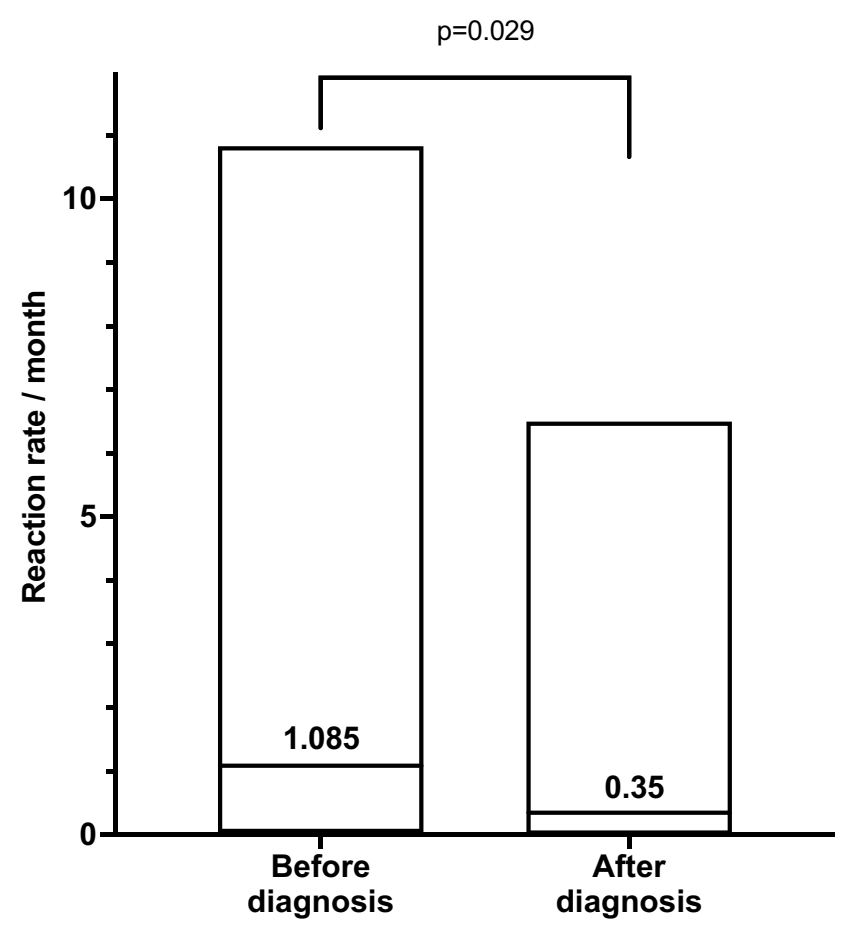

Figure I Allergic reaction rates before, and after, diagnosis among 43 individuals with omega-5-gliadin allergy.

\section{Adopted Treatment Strategies (Table 2)}

Twenty respondents (47\%) reported adherence to stringent recommended strategies. Seventeen respondents $(40 \%)$ practised a more liberal strategy than advised, mostly foregoing avoidance of wheat/gluten. Three respondents practised more stringent strategies than advised. Complete responses for adopted treatment strategies were missing for three individuals.

\section{Reactions After Diagnosis}

The mean reaction rate after diagnosis was significantly lower than that before diagnosis $(0.35$ per month vs 1.085 reactions per month, $\mathrm{p}=0.029$, Figure 1$)$. Among respondents, the range of allergic reaction rates was extremely wide, from zero to 6.5 per month. After diagnosis, the rate of reported allergic reactions per month was lower in 34 respondents $(79 \%), 10(23 \%)$ of whom experienced no reactions.

Reactions following diagnosis were attributed to various cofactors including unexpected exertion (15 respondents, $35 \%$ ); intentional gluten ingestion (13 respondents, 30\%); eating away from home (12 respondents, $28 \%$ ), and accidental gluten ingestion (11 respondents, 26\%) (Figure 2).

\section{Reactions After Diagnosis According to Treatment Strategy}

Respondents who adopted a stringent management strategy $(n=33,77 \%)$, with a combination of either complete

\section{Factors contributing to reactions}

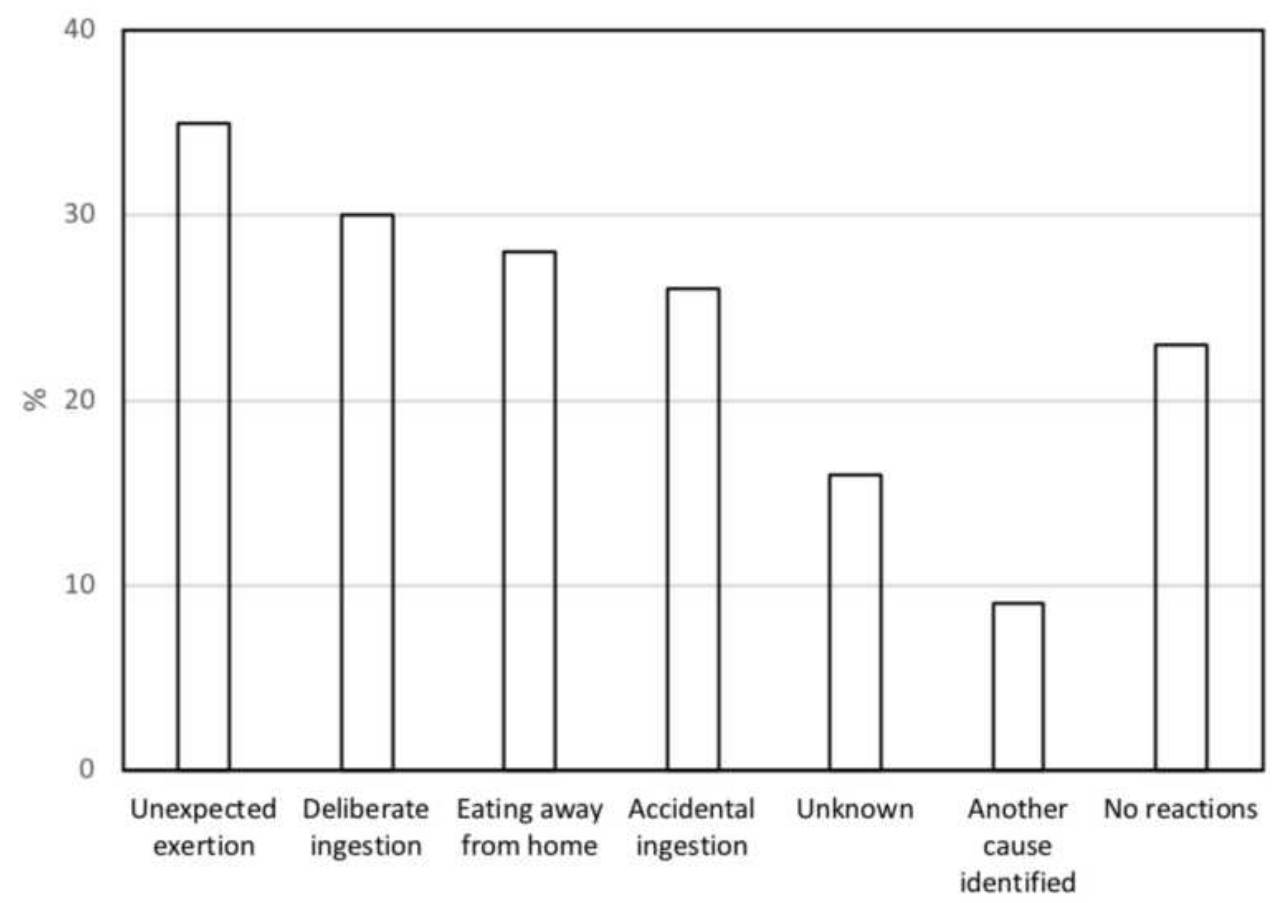

Figure 2 Proportion of individuals who have reported these risk factors as being associated with allergic reactions following diagnosis of omega-5-gliadin allergy. 


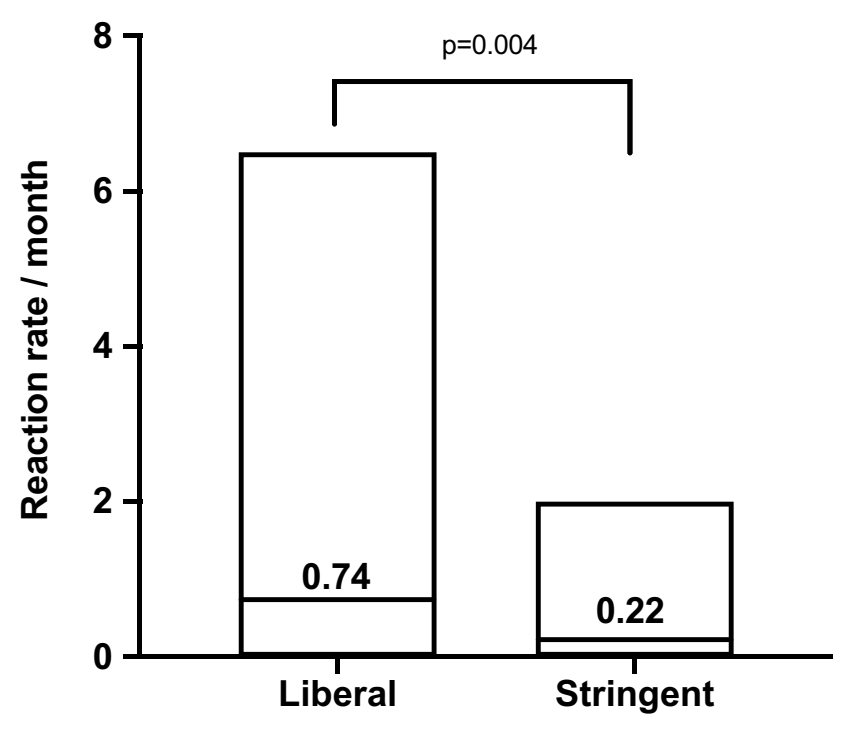

Figure 3 Allergic reaction rates following diagnosis of omega-5-gliadin allergy in 43 individuals stratified by adopted management approach. Stringent refers to complete avoidance of wheat/gluten and/or separation of wheat ingestion and exercise by 4 hours. Liberal refers to neither.

avoidance of dietary wheat/gluten ("avoiders") and/or strict temporal separation of food intake and exercise ("separators") had significantly lower reaction rates than respondents who practised a liberal management strategy $(n=10,23 \%)$, ie, those who were neither "avoiders" nor "separators" ( 0.22 per month vs 0.74 per month, $\mathrm{p}=0.004$, Figure 3 ). Reaction rate data were unavailable for one respondent.

When respondents were stratified by dietary strategy alone, reaction rates were not statistically different, although there was a trend towards a lower rate of reactions among the eight respondents (19\%) who completely avoided dietary wheat/gluten ("avoiders"), (0.09 per month vs 0.4 per month among "non-avoiders").

When respondents were stratified by exercise strategy alone, reaction rates again were not significantly different.

Reaction rates were not significantly influenced by dietitian consultation, age, sex, or previous anaphylaxis.

\section{Discussion}

In this study, capturing real-world experience as reported by patients with confirmed O-5-G allergy due to sensitization to the major associated wheat allergen O-5-G, a substantial reduction in allergic reaction rates occurred once a firm diagnosis was achieved. Both avoidance of wheat/gluten ingestion and temporal separation of ingestion from exercise were effective management strategies. Unfortunately, as is frequently the case in clinical practice, many patients in our cohort did not adopt the recommended strategies. Our data also endorse O-5-G allergy as an epidemiologically distinct food allergy.

The clinical syndrome of WDEIA has long been recognized, but its diagnosis often remains elusive to those unfamiliar with the condition. Challenges in diagnosis relate to the unique and counterintuitive clinical features, such that the individual may frequently eat wheat/gluten without any ill-effects. We accepted all positive results, noting that there is variability but overall persistence of O-5-G IgE over time. ${ }^{8}$ The ability to establish a definitive diagnosis with measurement of serum-specific IgE directed against $\mathrm{O}-5-\mathrm{G}$ has been available for many years ${ }^{7}$ yet median time to diagnosis in our cohort remained elevated at 2 years and $30 \%$ remained undiagnosed for 5 years, during which patients continued to experience many reactions, some of which were life-threatening. Reassuringly, once a diagnosis was made, patients reported a profound reduction in the rate of reactions, by approximately twothirds. These findings show the benefit of an early diagnosis and highlight the pressing need to shorten the patient's journey to achieving definitive diagnosis.

Following a diagnosis of $\mathrm{O}-5-\mathrm{G}$ allergy, a range of management choices regarding diet and exercise are open to individuals. We documented a variety of recommendations given to, and adopted by, our patient cohort. The majority (37 individuals, 88\%) recalled being recommended a stringent approach (either to avoid wheat/gluten completely, or to separate wheat/gluten ingestion and exercise by 4 hours), but disappointingly, only 20 individuals $(46 \%)$ adopted this recommendation. An additional three adopted a stringent approach despite not recalling such recommendations from their doctors. Although it was noted by many doctors that the patients did not have coeliac disease per se, adoption and explanation of a coeliac or gluten-free diet is more manageable socially for patients as most food labelling and purchased foods specify gluten content. Importantly, individuals who adopted a stringent approach experienced fewer reactions than those who adopted a liberal approach (neither avoiding wheat/gluten nor separating ingestion from exercise). The threshold of gluten and cofactors sufficient to provoke symptoms, however, may vary widely between individuals ${ }^{9,10}$ and further contribute to the difficulty in establishing both the diagnosis and optimal management.

There were too few respondents in our cohort to determine if one component of the stringent strategy was more effective than the other, ie avoidance versus separation. Other 
observational studies have found both strategies to be effective. ${ }^{6}$ In our cohort, unexpected exertion and accidental wheat/gluten ingestion (often associated with eating away from home) were both important factors associated with reactions occurring after diagnosis, so risks exist with both approaches. Intriguingly, it is possible that deliberate and regular consumption of wheat may raise the gluten ingestion threshold to develop subsequent exercise-triggered allergic reactions. ${ }^{11}$ We surmise that the ideal stringent approach for an individual should be tailored to that individual's circumstances, including their specific risks of unexpected exertion or accidental ingestion. Individuals who choose not to avoid wheat/gluten should be carefully counselled regarding the challenges of separating ingestion from exercise. Individuals who rely on wheat/gluten avoidance rather than separation from exercise should be provided training to avoid accidental ingestion, ideally through consultation with a dietitian (which rarely occurred in our cohort). We did not specify additional grains besides wheat in this cohort although reactions following, for example, rye flour ingestion has also been reported. ${ }^{12}$

The severity of allergic reaction of each episode has been shown to vary, ${ }^{13}$ although in our cohort the majority $(88 \%)$ reported multisystem anaphylaxis at least once. This appears to be higher than previously reported rates of $60-76 \%, 6,13$ and in our practice justifies the routine ongoing provision of an epinephrine-auto-injector.

Our respondents with O-5-G allergy had predominantly adult disease onset and relatively few atopic comorbidities, adding to similar findings reported by others. ${ }^{6,13-15}$ The atopic march, and rates of onset and remission of specific food allergies in childhood are well characterized, ${ }^{16}$ as are relevant in utero and early childhood factors that influence the onset of such IgE mediated food allergy. However, these are probably far less relevant to adult-onset food allergy such as O-5-G allergy. The identification of alternative risk factors and mechanisms of disease pathogenesis may be required to facilitate prevention and alter the natural history of this condition. Other determinants of disease such as ethnicity were not assessed in our study although may in fact be relevant in the development or recognition of the condition. ${ }^{17}$

Our study, based on respondent self-report, was able to establish neither the true rigor of wheat/gluten avoidance nor separation of ingestion from exercise. Furthermore, dietary and lifestyle adherence may alter over time and we acknowledge this may have an undetermined effect on allergic reaction rate over time. Consultation with a dietitian, necessary to optimize wheat/gluten avoidance, was infrequent in our cohort. Respondents who only avoided wheat were not analyzed separately from those who also avoided other glutencontaining grains (rye, barley and oats), whose relevance to $\mathrm{O}-5-\mathrm{G}$ allergy is unclear. Our case definition requiring positive O-5-G serum-specific IgE may have excluded individuals with WDEIA due to other gluten components. The intensity of exercise to trigger WDEIA varies between individuals and between occasions in the same individual, so self-reports of separating ingestion from exercise are highly subjective. Reaction co-factors such as NSAIDs and aspirin, alcohol, and viral infection are relevant to WDEIA $^{18}$ but difficult to quantify or identify retrospectively, and were not examined in this survey.

\section{Conclusion}

$\mathrm{O}-5-\mathrm{G}$ allergy is a unique clinical problem requiring the ingestion of wheat as well as the presence of a co-factor for an allergic reaction to occur. It is additionally distinct in that it is largely adult in onset and is associated with lower rates of atopic co-morbidities than other food allergies. Accurate diagnosis of O-5-G allergy is fundamental to improving the clinical trajectory. Patients are usually recommended a stringent management approach, but many do not adhere to such strategies. Those who do, however, enjoy significantly lower reaction rates than those who do not. We had insufficient respondents to support recommending dietary avoidance of gluten over separating ingestion from exercise, or vice versa, and so recommend tailoring advice to the individual. Future prospects for disease modification require greater understanding of the unique disease mechanisms.

\section{Author Contributions}

All authors contributed to data analysis, drafting or revising the article, have agreed on the journal to which the article will be submitted, gave final approval of the version to be published, have agreed on the journal to which the article has been submitted, and agree to be accountable for all aspects of the work.

\section{Funding}

There is no funding to report.

\section{Disclosure}

$\mathrm{MH}$ has received grants-in-aid, speaker fees, and fees for serving on the advisory boards of GlaxoSmithKline, AstraZeneca, Novartis, Teva, Sanofi, and Seqirus, all paid to his institutional employer Alfred Health. Interim Data presented in Poster Format at EAACI Barcelona 2015. The remaining authors report no conflicts of interest in this work. 


\section{References}

1. Pastorello E, Farioli L, Stafylaraki C, et al. Wheat-dependent exercise-induced anaphylaxis caused by a lipid transfer protein and not by Omega-5-gladin. Ann Allergy Asthma Immunol. 2014;112 (4):386-387. doi:10.1016/j.anai.2014.01.012

2. Scherf KA, Brockow K, Biedermann T, et al. Wheat-dependent exercise-induced anaphylaxis. Clin Exp Allergy. 2015;46(1):10-20. doi:10.1111/cea. 12640

3. Zhu YQ, Wang DQ, Liu B, et al. Wheat-dependent exercise-induced anaphylaxis in Chinese people: a clinical research on 33 cases with antigenic analysis of wheat proteins. Clin Exp Dermatol. 2019;45 (1):56-62. doi:10.1111/ced.14035

4. Brockow K, Kneissl D, Valentini L, et al. Using a gluten oral food challenge protocol to improve diagnosis of wheat-dependent exercise-induced anaphylaxis. J Allergy Clin Immunol. 2015;135 (4):977-988. doi:10.1016/j.jaci.2014.08.024

5. Kushimoto H, Aoki T. Masked type I wheat allergy. Arch Dermatol. 1985;121(3):355-360. doi:10.1001/archderm.1985.01660030077023

6. Kennard L, Thomas I, Rutlowski K, et al. A multicenter evaluation of diagnosis and management of omega- 5 gliadin allergy (also known as wheat-dependent exercise-induced anaphylaxis) in 132 adults. J Allergy Clin Immunol Pract. 2018;6(6):1892-1897. doi:10.1016/j. jaip.2018.02.013

7. Matsuo H, Morita E, Tatham AS, et al. Identification of the IgEbinding Epitope in $\omega-5$ Gliadin, a major allergen in wheat-dependent exercise-induced anaphylaxis. $J$ Biol Chem. 2004;279 (13):12135-12140. doi:10.1074/jbc.M311340200

8. Hamada Y, Chinuki Y, Fukutomi Y, et al. Clinical communications: long-term dynamics of omega-5 gliadin-specific IgE levels in patients with adult-onset wheat allergy. J Allergy Clin Immunol Pract. 2020;8 (3):1149-1151. doi:10.1016/j.jaip.2019.10.008

9. Christensen MJ, Eller E, Mortz CG, Brockow K, Bindslev-Jensen C. Exercise lowers threshold and increases severity, but wheat-dependent, exercise-induced anaphylaxis can be elicited at rest. J Allergy Clin Immunol Pract. 2018;6(2):514-520. doi:10.1016/j.jaip.2017.12.023
10. Brockow K, Kneissl D, Valentini L, et al. Using a gluten oral food challenge protocol to improve diagnosis of wheat-dependent exercise-induced anaphylaxis. J Allergy Clin Immunol. 2014:1-12.

11. Christensen MJ, Eller E, Mortz CG, Brockow K, Bindslev-Jensen C. Clinical and serological follow-up of patients with WDEIA. Clin Transl Allergy. 2019;1-4. doi:10.1186/s13601-019-0243-1

12. Garcia-Menaya JM, Chiarella GM, Cordobés-Durán C, Mahecha AC, Bobadilla-González P. Rye-dependent exercise-induced anaphylaxis. Ann Allergy Asthma Immunol. 2016;117(5):566-568. doi:10.1016/j. anai.2016.08.032

13. Le TA, Al Kindi M, Tan JA, et al. The clinical spectrum of omega-5-gliadin allergy. Intern Med J. 2016;46(6):710-716. doi:10.1111/imj.13091

14. Wong GKY, Huissoon AP, Goddard S, Collins DM, Krishna MT. Wheat dependent exercise induced anaphylaxis: is this an appropriate terminology? J Clin Pathol. 2010;63(9):814-817. doi:10.1136/ jcp.2010.078808

15. Kamdar T, Peterson S, Lau C, Saltoun C, Gupta R, Bryce PJ. Clinical communications: prevalence and characteristics of adult onset food allergy. $J$ Allergy Clin Immunol Pract. 2015;3(1):114-115. doi:10.1016/j.jaip.2014.07.007

16. Sicherer SH, Sampson HA. Food allergy: a review and update on epidemiology, pathogenesis, diagnosis, prevention, and management. $J$ Allergy Clin Immunol. 2018;141(1):41-58. doi:10.1016/j. jaci.2017.11.003

17. Li PH, Thomas I, Chi-yan wong J, Rutkowski K, Lau CS. Differences in omega-5-gliadin allergy: East versus West. Asia Pac Allergy. 2020;10(1). PMID: 32099827. doi:10.5415/apallergy.2020.10.e5

18. Christensen MJ, Eller E, Mortz CG, Brockow K, Bindslev-Jensen C. Wheat-dependent cofactor-augmented anaphylaxis: a prospective study of exercise, aspirin, and alcohol efficacy as cofactors. J Allergy Clin Immunol Pract. 2019;7(1):114-121. doi:10.1016/j. jaip.2018.06.018
Journal of Asthma and Allergy

\section{Publish your work in this journal}

The Journal of Asthma and Allergy is an international, peer-reviewed open-access journal publishing original research, reports, editorials and commentaries on the following topics: Asthma; Pulmonary physiology; Asthma related clinical health; Clinical immunology and the immunological basis of disease; Pharmacological interventions and

Submit your manuscript here: https://www.dovepress.com/journal-of-asthma-and-allergy-journa

\section{Dovepress}

new therapies. The manuscript management system is completely online and includes a very quick and fair peer-review system, which is all easy to use. Visit http://www.dovepress.com/testimonials.php to read real quotes from published authors. 